\title{
ATIVIDADE FOTOCATALÍTICA DE NANOFIOS DE TIO 2 NA DEGRADAÇÃO DO CORANTE AZUL DE METILENO SOB LUZ UV
}

\author{
M.O. HONÓRIO ${ }^{1}$, A. MENDES ${ }^{2}$, L. ANDRADE ${ }^{2}$ e R.F.P.M.MOREIRA ${ }^{1}$ \\ ${ }^{1}$ Universidade Federal de Santa Catarina, Departamento de Engenharia Química e Engenharia de \\ Alimentos \\ ${ }^{2}$ LEPABE - Faculdade de Engenharia, Universidade do Porto, rua Dr. Roberto Frias, 4200-465 \\ Porto, Portugal \\ E-mail para contato: micheline@fe.up.pt
}

\begin{abstract}
RESUMO - A oxidação fotocatalítica vem se mostrando promissora na degradação de diversos contaminantes. Porém, sua viabilidade econômica em larga escala implica a utilização de catalisadores mais estáveis e que possam ser ativados tanto por radiação UV quanto visível. Portanto, a redução do bandgap com correspondente deslocamento da zona de absorção para o visível permitirá que as reações fotocatalíticas com $\mathrm{TiO}_{2}$ sejam eficientes no visível. A dopagem do $\mathrm{TiO}_{2}$ com não-metais e o controle da morfologia são estratégias bem sucedidas para reduzir o bandgap e permitir fotoatividade no visível. Neste estudo, avaliou-se a fotoatividade de nanofios de $\mathrm{TiO}_{2}$ sintetizados via rota hidrotérmica. A morfologia foi identificada por MEV, o bandgap, quantificado por análise de reflectância difusa e a fotoatividade sob luz UV, por degradação do azul de metileno em solução aquosa. Os estudos revelaram a presença de nanofios e, quando dopados com nitrogênio revelaram redução do bandgap $(\approx 5 \%)$ e da fotoatividade.
\end{abstract}

\section{INTRODUÇÃO}

Dióxido de titânio $\left(\mathrm{TiO}_{2}\right)$ é um material semicondutor amplamente utilizado (Asiah et al., 2013; Khalid et al., 2012; Lv et al., 2012; Min et al., 2013; Yang et al., 2010) em vários campos de pesquisa, como é o caso da fotocatálise, especificamente fotoinativação de microrganismos e fotoabatimento de poluentes, a produção de hidrogênio via fotohidrólise e células solares sensibilizadas por corante (DSCs). (Shao et al., 2010; Tian et al., 2013)

A versatilidade de aplicações do $\mathrm{TiO}_{2}$ pode ser explicada por suas propriedades únicas (nãotoxicidade, excelente eficiência de conversão de energia e boa estabilidade química) e o fato de ser prontamente disponível e relativamente barato. Além disso, o $\mathrm{TiO}_{2}$ mostra bandas bem posicionadas, exibindo poder de oxidação forte à temperatura e pressão ambiente $(3,0 \mathrm{~V})$ fazendo com que ativação fotocatalítica ocorra, somente, sob a luz UV (5 \% da radiação solar incidente na superfície da Terra) e seja muito baixa sob a luz visível (45 \% da radiação solar incidente na superfície da Terra) (Niu et al., 2014). Por outro lado, os elétrons fotogerados são capazes de reduzir oxigênio a superóxido (-0,2 V). 
Estudos recentes têm sugerido que os nanofios de $\mathrm{TiO}_{2}$ favorecem o transporte de cargas ao longo da dimensão longitudinal, para além de diminuírem a taxa de recombinação elétron-lacuna (Shao, Sun et al, 2010; Lv, Zhang et al, 2012; Tian, Pan et al, 2013). Além disso, a dopagem do $\mathrm{TiO}_{2}$ com nitrogênio tem favorecido o estreitamento do bandgap do $\mathrm{TiO}_{2}$ aumentando a absorção no espectro da luz visível, crucial para melhorar o desempenho fotocatalítico do $\mathrm{TiO}_{2}$. (Asahi et al., 2001; Niu et al., 2014; Yang et al., 2010)

Nanofios de $\mathrm{TiO}_{2}$ dopados com nitrogênio podem ser preparados pelo processo sol-gel, método solvotérmico ou hidrotérmico utilizando como precursor $\mathrm{TiO}_{2}$ comercial P25 (He et al., 2011; Yang et al., 2010; Yu et al., 2009). Neste trabalho, avaliou-se a atividade fotocatalítica em estudos de cinética de degradação do corante azul de metileno, utilizando nanofios de $\mathrm{TiO}_{2}$ (dopados com nitrogênio e não dopados), que foram sintetizados pelo método hidrotérmico.

\section{Material e Métodos}

\subsection{Material}

$\mathrm{TiO}_{2}$ comercial (P25) foi obtido da Evonik. Hidróxido de sódio ( $\mathrm{NaOH}, 98 \%$ de pureza), trietilamina (TEA, $98 \%$ de pureza) e corante azul de metileno (AM) foram adquiridos da SigmaAldrich $\mathrm{O}$ ácido clorídrico $(\mathrm{HCl}, 37 \%$ grau analítico) foi fornecido pela Fisher Scientific e etanol anidro (99,5\% em volume) pela Aga.

\subsection{Síntese dos nanofios}

Amostras de $\mathrm{TiO}_{2}$ dopadas com nitrogênio $\left(\mathrm{N}-\mathrm{TiO}_{2}\right)$ foram sintetizadas pelo método hidrotérmico. A solução percursora foi preparada adicionando $1,0 \mathrm{~g}$ de $\mathrm{TiO}_{2}$ (P25 da Evonik, Alemanha) a $100 \mathrm{~mL}$ de uma solução aquosa de hidróxido de sódio $10 \mathrm{M}$. Em seguida, diferentes quantidades de TEA (2 e $4 \mathrm{~mL}$ ) foram adicionadas à solução para obtenção de diferentes níveis de dopagem e agitou-se por $1 \mathrm{~h}$. A solução final foi então transferida para uma autoclave de Teflon ${ }^{\circledR}$ com um encamisamento de aço inox e tratada a $180{ }^{\circ} \mathrm{C}$ por um tempo específico (os experimentos foram realizados por $72 \mathrm{~h}$ e 96 h). Após reação, a autoclave foi resfriada a temperatura ambiente. O pó de $\mathrm{TiO}_{2}$, com e sem dopagem de nitrogênio, foi obtido depois da filtração e lavagem com água destilada e ácido clorídrico $(\mathrm{HCl}, \mathrm{pH} 2)$ várias vezes até $\mathrm{pH} 7$ ser atingido; posteriormente o pó foi seco a $60^{\circ} \mathrm{C}$ por $24 \mathrm{~h}$. O material resultante foi calcinado a $550{ }^{\circ} \mathrm{C}$ ou $700{ }^{\circ} \mathrm{C}$ (a uma taxa de aquecimento de 2 $\left.{ }^{\circ} \mathrm{C} / \min \right)$.

Duas quantidades diferentes de TEA e duas temperaturas de calcinação foram consideradas. Assim, as amostras correspondentes foram nomeadas de acordo com a sua composição de TEA e tempo de reação e temperatura de calcinação (Tabela 1). $\mathrm{TiO}_{2} \mathrm{P} 25$ comercial foi usado como referência para todos os tratamentos referência para todos os tratamentos. 
Tabela 1 - Fotocatalisadores sintetizados - condições experimentais utilizadas

\begin{tabular}{cccc}
\hline Fotocatalisador & $\%$ TEA & Tempo de reação $(\mathrm{h})$ & Temperatura de calcinação $\left({ }^{\circ} \mathrm{C}\right)$ \\
\hline NF- 72-700 & 0 & 72 & 700 \\
NF- 96-700 & 0 & 96 & 700 \\
NF-72-550 & 0 & 72 & 550 \\
NF-N(2)-72-700 & 2 & 72 & 700 \\
NF-N(2)-96-700 & 2 & 96 & 700 \\
NF-N(4)-72-700 & 4 & 72 & 700 \\
NF-N(4)-96-700 & 4 & 96 & 700 \\
\hline
\end{tabular}

\subsection{Caracterização}

A morfologia das amostras sintetizadas foi avaliada usando microscópio eletrônico de varredura (MEV): JEOL JSM 6301F / Oxford INCA Energy 350. Reflectância difusa foi medida na faixa de 240 - $800 \mathrm{~nm}$ usando um espectrofotômetro UV 3600 (Shimadzu) para determinar a energia de bandgap $\left(E_{g}\right)$. Análise termogravimétrica foi realizada em um analisador TG 209 FI (Netzch) Iris ${ }^{\circledR} \mathrm{e}$ foi realizada a partir de $30^{\circ} \mathrm{C}$ até $990^{\circ} \mathrm{C}$ a uma taxa de aquecimento de $2^{\circ} \mathrm{C} / \mathrm{min}$, num fluxo de $\mathrm{N}_{2}$ de $30 \mathrm{~mL} / \mathrm{min}$.

\subsection{Atividade fotocatalítica}

A atividade fotocatalítica das diferentes amostras foi estimada por meio da degradação do corante azul de metileno em um reator com lâmpada UV como fonte de radiação (irradiância da superfície de mistura de $10 \mathrm{~W} / \mathrm{m}^{2}$ ). Para os experimentos fotocatalíticos, foi adicionado $50 \mathrm{mg}$ de fotocatalisador em $100 \mathrm{~mL}$ de azul de metileno (AM) com concentração de $10 \mathrm{mg} / \mathrm{L}$ num béquer. A suspensão foi mantida sob agitação magnética por 30 min sem exposição à luz UV. Em seguida, a reação fotocatalítica foi iniciada quando a solução foi colocada sob a luz UV. Depois a absorbância foi determinada em $665 \mathrm{~nm}$.

\section{RESULTADOS E DISCUSSÃO}

\subsection{Caracterização}

As estruturas dos nanofios foram analisadas por MEV. As Figuras 1 e 2 mostram as morfologias dos nanofios sintetizados após 72 h e 96 h, com e sem adição de TEA, respectivamente. 


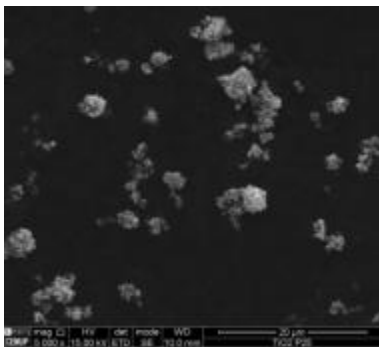

(a) P25

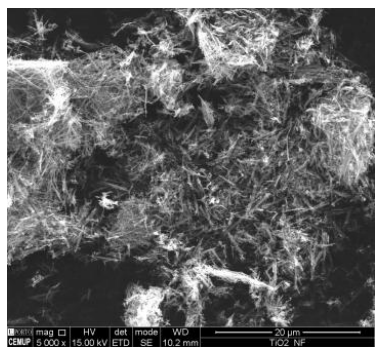

(b) NF-72-700

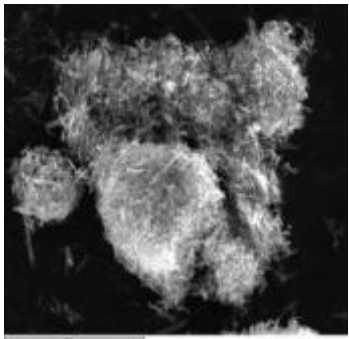

(c) NF-96-700

Figura 1 - Imagens de MEV do $\mathrm{TiO}_{2}$ sintetizado sem TEA a diferentes tempos de síntese: a) P25; b) tempo de reação de $72 \mathrm{~h}, 700{ }^{\circ} \mathrm{C}$; c) tempo de reação de $96 \mathrm{~h}, 700{ }^{\circ} \mathrm{C}$.

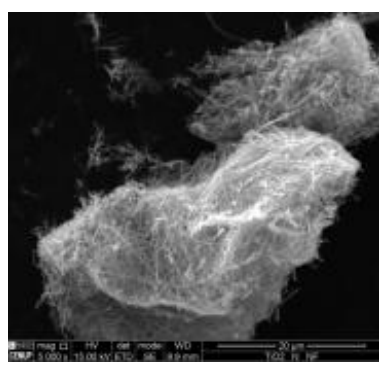

(a) $\mathrm{NF}-\mathrm{N}(2)-72-700$

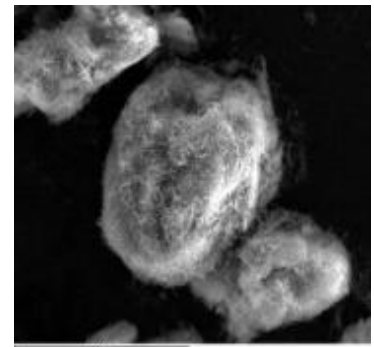

(b) $\mathrm{NF}-\mathrm{N}(2)-96-700$

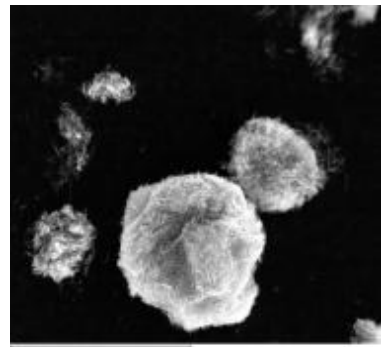

(c) $\mathrm{NF}-\mathrm{N}(4)-72-700$

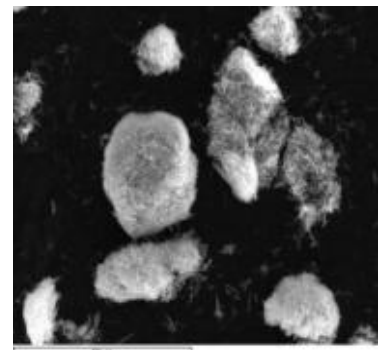

(d) $\mathrm{NF}-\mathrm{N}(4)-96-700$

Figura 2 - Imagens de MEV do $\mathrm{TiO}_{2}$ sintetizado com diferentes \% TEA e diferentes tempos de síntese: a) 2 vol.\% de TEA e tempo de reação de 72 h; b) 2 vol.\% de TEA e tempo de reação de 96 h;

c) 4 vol.\% de TEA tempo de reação de 72 h; d) 4 vol.\% de TEA tempo de reação de 96 h.

Independente dos parâmetros de operação (tempo de síntese, percentagem de TEA e temperatura de calcinação) observa-se que em todas as amostras há a presença de nanofios e a formação de aglomerados. Em particular, existe uma tendência de ter um crescimento no tamanho dos aglomerados quando o tempo de síntese é maior, temperatura de calcinação é menor e quando $4 \%$ de TEA é adicionado à amostra; isto pode ocorrer uma vez que estas condições operacionais conduzem à formação de nanofios mais longos.

Na Figura 3 tem-se representadas as morfologias dos nanofios sintetizados após $72 \mathrm{~h}$ sem adição de TEA e calcinados a $700{ }^{\circ} \mathrm{C}$ e $550^{\circ} \mathrm{C}$. 


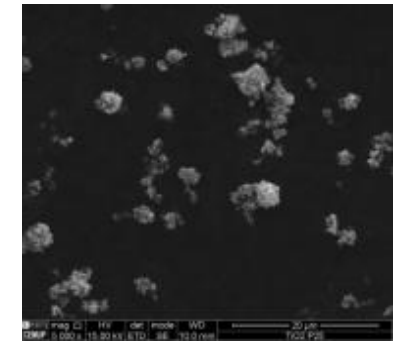

(a) P25

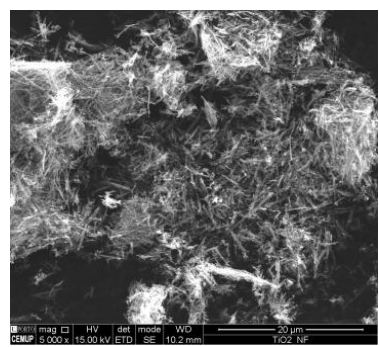

(b) NF-72-700

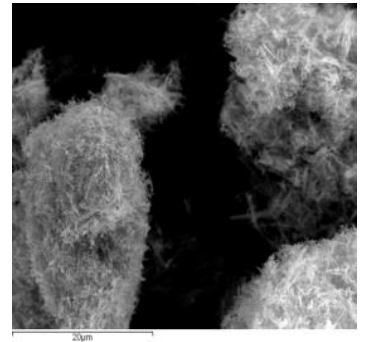

(c) NF-72-550

Figura 3 - Imagens de $\mathrm{MEV}$ do $\mathrm{TiO}_{2}$ sintetizado sem TEA por $72 \mathrm{~h}$ a diferentes temperaturas de calcinação: a) P25; b) tempo de síntese, $700{ }^{\circ} \mathrm{C}$; c) tempo de síntese, $550{ }^{\circ} \mathrm{C}$.

Análise termogravimétrica (TG) - Figura $4 \mathrm{a}$ - mostra que os nanofios sintetizados durante $72 \mathrm{~h}$ e com 4 vol.\% de TEA apresentaram maior perda de massa ( $8 \%$ ), enquanto os nanofios sintetizados durante 96 h apresentaram a menor - Figura 4b - estando de acordo com a literatura. (Asiah et al., 2013; Daya Mani et al., 2013; Min et al., 2013) A curva do TGA mostrou que entre $30-400{ }^{\circ} \mathrm{C}$ a perda de massa pode ser atribuída à perda de água e decomposição da matéria orgânica. Acima de $400{ }^{\circ} \mathrm{C}$ até $990{ }^{\circ} \mathrm{C}$ está associada à combustão da matéria orgânica, sendo a maior perda de massa verificada para os nanofios sintetizados durante 72 h e com 4 vol.\% de TEA.

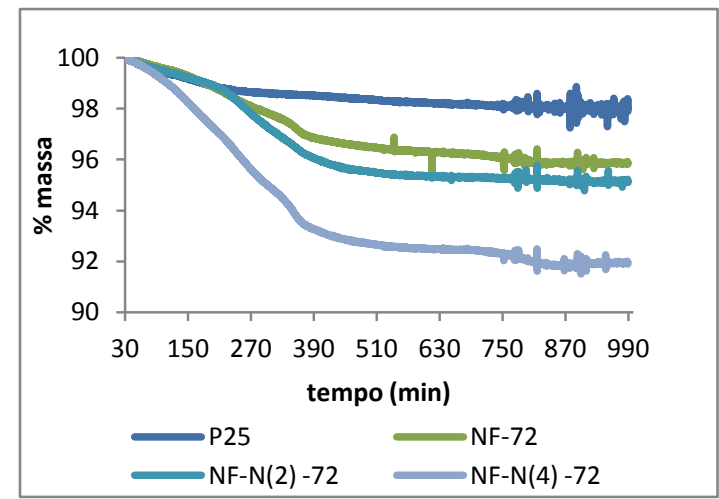

(a)

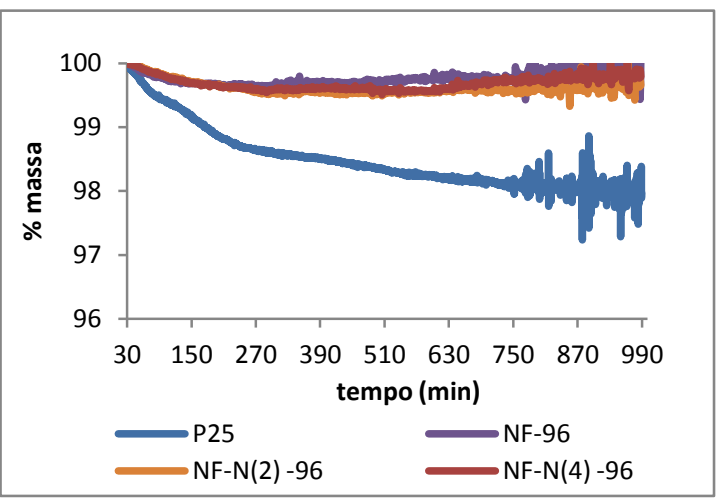

(b)

Figure 4 - Comparação das análises de TG dos nanofios sintetizados com P25 e diferentes percentagens de TEA e tempos de reação: a) tempo de reação de 72 h e temperatura de calcinação de $700{ }^{\circ} \mathrm{C}$; b) tempo de reação de 96 h e temperatura de calcinação de $700{ }^{\circ} \mathrm{C}$.

Nota-se que nanofios sintetizados a $550{ }^{\circ} \mathrm{C}$, além de possuírem aglomerados maiores (MEV) apresentaram pouca ou nenhuma perda de massa quando comparados com o P25 (inferior a 2\%) seguido pelos nanofios calcinados a $700^{\circ} \mathrm{C}(4 \%)$. 


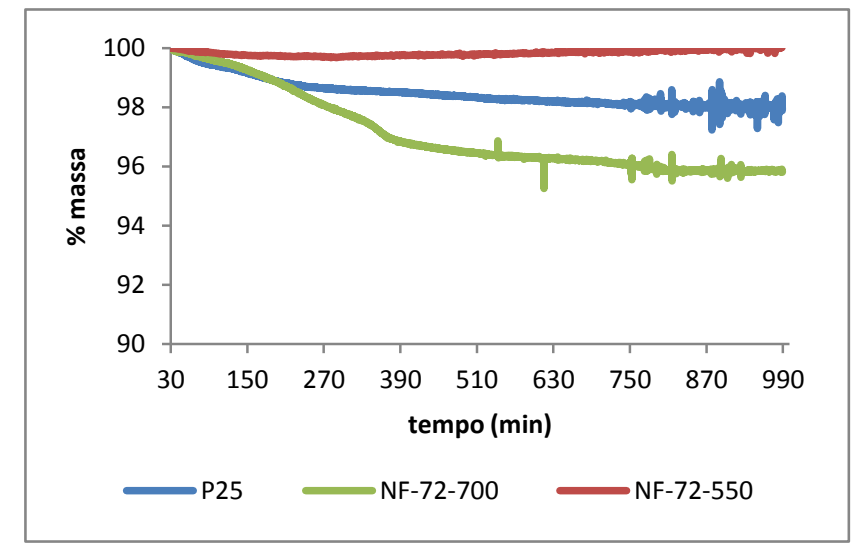

Figure 5 - Comparação das análises de TG dos nanofios, sintetizados durante $72 \mathrm{~h}$ e diferentes temperaturas de calcinação $\left(700{ }^{\circ} \mathrm{C}\right.$ e $\left.550{ }^{\circ} \mathrm{C}\right)$, com P25.

A determinação do bandgap $E g$ foi estimada por meio da reflectância difusa $(R)$ na faixa de 240 - $800 \mathrm{~nm}$ e pela aplicação da função de Kubelka-Munk $(\mathrm{KM}=\mathrm{F}(R))$, plotando $(\mathrm{KM} \times \mathrm{h} v)^{1 / 2}$ em função da energia (em eV). Os resultados estão apresentados na Figura 6. Nenhuma diferença na energia de bandgap dos fotocatalisadores com diferentes níveis de dopagem foi observada quando sintetizados por $96 \mathrm{~h}$ e temperatura de calcinação de $700^{\circ} \mathrm{C}$. No entanto, os nanofios sintetizados por 72 horas e $4 \%$ de TEA, os quais apresentaram menor comprimento, apresentaram uma maior redução (5\%) no bandgap quando comparados com o P25.

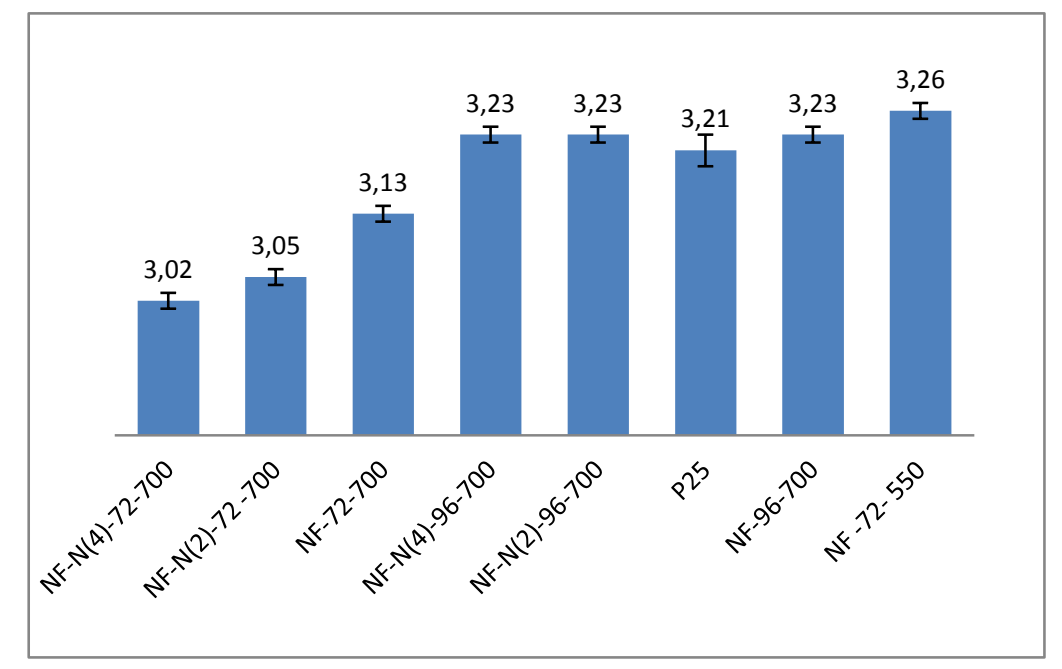

Figura 6 - Energia de bandgap (eV) estimada para o P25 e nanofios sintetizados 


\subsection{Atividade Fotocatalítica}

O corante azul de metileno (AM) foi utilizado como poluente orgânico para avaliar a atividade fotocatalítica dos nanofios de $\mathrm{TiO}_{2}$ dopado. Na Figura 7 é apresentada a atividade fotocatalítica sob luz UV para a degradação do $\mathrm{AM}$ dos fotocatalisadores de $\mathrm{TiO}_{2}$ sintetizados com diferentes quantidades de TEA e durante diferentes tempos de síntese.

Os nanofios não-dopados e os dopados com $96 \mathrm{~h}$ de tempo de reação apresentaram eficiência de remoção do azul de metileno similar ao P25 sob luz UV $(\approx 90 \%)$. Em relação às amostras de nanofios preparadas com $72 \mathrm{~h}$ de tempo de reação (NF-72, NF-N (2) -72 e de NF-N (4) -72), notou-se que apresentou menor eficiência de degradação fotocatalítica, em torno de $50 \%$, o que é explicado pela redução da energia do bandgap.

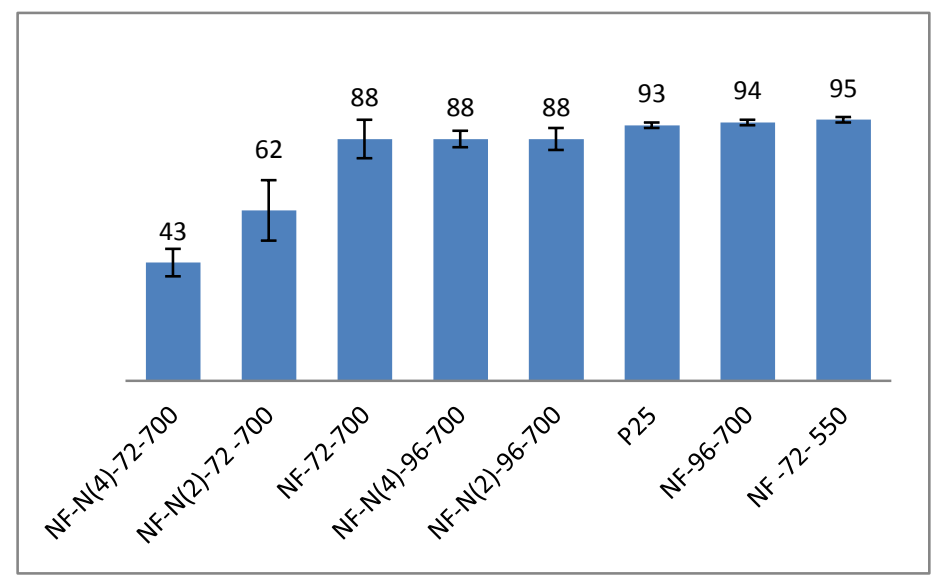

Figura 7. Eficiência de degradação (\%) do corante azul de metileno sob luz UV usando $\mathrm{TiO}_{2}$ sintetizados, com e sem TEA por diferentes tempos de síntese, medidos por meio da redução de absorbância em 665 nm após 4 h, comparados ao P25.

\section{CONCLUSÃO}

Neste trabalho nanofios de $\mathrm{TiO}_{2}$ foram sintetizados pelo método hidrotérmico usando P25 como precursor. Foram utilizados diferentes tempos de preparação e diferentes quantidades de TEA (confirmado por MEV). A energia de bandgap dos nanofios foi estimada com base na reflectância difusa; bandgaps entre 3,02 e 3,13 eV foram obtidos para $72 \mathrm{~h}$ de tempo de reação. $\mathrm{O}$ valor mais baixo de bandgap foi obtida para 4 vol.\% de TEA. Esta redução do bandgap foi de fato refletida em menores valores de eficiência da degradação AM sob luz UV $(\approx 50 \%)$, ou seja, na atividade catalítica sob luz UV indicando que estes nanofios possam ter potencial para fotodegradar o corante azul de metileno sob luz visível. 


\section{REFERENCIAS}

ASAHI, R., MORIKAWA, T., OHWAKI, T., AOKI, K., TAGA, Y. Visible-Light Photocatalysis in Nitrogen-Doped Titanium Oxides. Science 293, 269-271.2001.

ASIAH, M.N., MAMAT, M.H., KHUSAIMI, Z., ACHOI, M.F., ABDULLAH, S., RUSOP, M., Thermal stability and phase transformation of $\mathrm{TiO}_{2}$ nanowires at various temperatures. M. Eng. 108, 134-137. 2013.

DAYA MANI, A., RAMA RAJU, B., XANTHOPOULOS, N., GHOSAL, P., SREEDHAR, B., SUBRAHMANYAM, C. Effect of fuels on combustion synthesis of TiO2 - Towards efficient photocatalysts for methylene blue oxidation and $\mathrm{Cr}$ (VI) reduction under natural sunlight. Chem. Eng. J. 228, 545-553. 2013.

HE, T., HU, Z.S., LI, J.L., YANG, G.W. Surface Effect and Band-Gap Oscillation of TiO 2 Nanowires and Nanotubes. The J. Phys.l Chem. C 115, 13837-13843. 2011.

KHALID, N.R., AHMED, E., HONG, Z., ZHANG, Y., AHMAD, M. Nitrogen doped TiO 2 nanoparticles decorated on graphene sheets for photocatalysis applications. Curr. Appl. Phys. 12, 1485-1492. 2012.

LV, X., ZHANG, H., CHANG, H.Improved photocatalytic activity of highly ordered $\mathrm{TiO}_{2}$ nanowire arrays for methylene blue degradation. Mat. Chem. Phys. 136, 789-795. 2012.

MIN, Y., HE, G., LI, R., ZHAO, W., CHEN, Y., ZHANG, Y. Doping nitrogen anion enhanced photocatalytic activity on $\mathrm{TiO}_{2}$ hybridized with graphene composite under solar light. Sep. Purif. Techn. 106, 97-104. 2013.

NIU, M., CHENG, D., CAO, D. Understanding the Mechanism of Photocatalysis Enhancements in the Graphene-like Semiconductor Sheet/ $\mathrm{TiO}_{2}$ Composites. The J. Phys. Chem. C 118, 5954-5960. 2014.

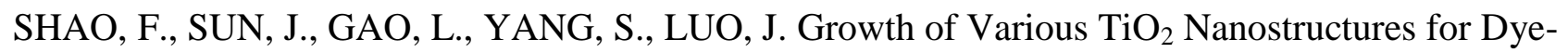
Sensitized Solar Cells. The J. Phys. Chem. C 115, 1819-1823. 2010.

TIAN, G., PAN, K., CHEN, Y., ZHOU, J., MIAO, X., ZHOU, W., WANG, R., FU, H. Vertically aligned anatase $\mathrm{TiO}_{2}$ nanowire bundle arrays: Use as Pt support for counter electrodes in dyesensitized solar cells. J. P. S. 238, 350-355. 2013.

YANG, G., JIANG, Z., SHI, H., XIAO, T., YAN, Z. Preparation of highly visible-light active Ndoped $\mathrm{TiO}_{2}$ photocatalyst. J. Mat. Chem. 20, 5301-5309. 2010.

YU, A., WU, G., ZHANG, F., YANG, Y., GUAN, N. Synthesis and Characterization of N-doped $\mathrm{TiO}_{2}$ Nanowires with Visible Light Response. Catal. Lett. 129, 507-512. 2009. 